\title{
Saisir les représentations sociales du rapport affectif aux lieux par la cartographie mentale
}

\author{
Thierry Ramadier, UMR SAGE, Strasbourg
}

L'objectif de cet atelier, dont la posture était surtout exploratoire, consistait, à mettre en évidence les dimensions émotionnelles du rapport à l'espace que nous partageons, en proposant une procédure de description collective d'un lieu à partir de la cartographie socio-cognitive. Le matériel utilisé était le Jeu de Reconstruction Spatiale (JRS), un outil utilisé autant dans le cadre de processus participatifs d'aménagement urbain que dans les enquêtes en sciences sociales sur le rapport à l'espace géographique. Cette technique d'enquête a la particularité de faciliter la comparaison entre les groupes sociaux (Ramadier et Bronner, 2006). Elle a permis, dans cet atelier et à partir d'une procédure originale que nous décrirons, de cerner dans quelle mesure le rapport affectif aux lieux n'est pas uniquement un rapport personnel à ces lieux, mais également un rapport qui est partagé, voire construit avec les autres. Ainsi, nous sommes partis de I'hypothèse qui guide mes travaux depuis quelques années (Ramadier et Moser, 1998, Ramadier, 2010, Dias et Ramadier, 2018) et selon laquelle les représentations spatiales sont des représentations sociales au sein desquelles les processus cognitifs à l'œuvre (lecture multidimensionnelle vs égocentrée de l'espace) dépendent de schèmes cognitifs (ou dispositions) préalablement acquis pour lire l'espace géographique en question. Ces schèmes étant fortement dépendant des ségrégations sociales dans l'espace (socialisation à l'espace), et par conséquent des positions sociales de la personne, c'est le modèle durkheimien de la correspondance entre les structures sociales et cognitives (Durkheim et Mauss, 1903), plutôt que le modèle culturaliste des significations différenciés de l'espace, qui est le support théorique sous-jacent à l'appréhension et à la compréhension du rapport à l'espace géographique des individus. Ce modèle permet ainsi de rendre compte d'un sentiment général de ne pas se sentir à sa place quand ce ajustement entre situation sociale et cognitive fait défaut, et du sentiment de se sentir à sa place (" d'être comme un poisson dans l'eau ») quand au contraire l'ajustement est bien présent. Autrement dit les représentations sociales de l'espace ne sont pas que des constructions cognitive. Ce sont également des représentations sociales construites à partir des rapports émotionnels à l'espace. Pour le dire autrement, dans cet atelier nous avons cherché à relever les dimensions sociales des rapports affectifs aux lieux. Et le principe méthodologique qui l'a guidé à consisté à cerner les convergences géographiques (ou polarisation spatiale) d'affects, à l'échelle d'un groupe, sur le terrain d'enquête.

\section{Description du déroulé de l'atelier}

Cet atelier a été programmé vers la fin du colloque de sorte à utiliser les lieux du colloque (le château et éventuellement ces pièces, les salles de travail, les différents lieux du jardin, etc.) comme terrain d'analyse et d'exploration de ces affects collectifs et/ou partagés.

La procédure proposée a été la suivante. Nous avons tout d'abord divisé les participants en trois, de sorte à constituer des groupes de travail inférieurs à 10 participants. Puis le déroulé de l'atelier était composé de trois étapes: 
Etape 1: la construction collective des lieux du colloque à l'aide du JRS, à partir de la consigne collective suivante :

"Tout d'abord Je vais vous demander de reconstruire collectivement et sur cette table les lieux du colloque dans lesquels nous vivons depuis vendredi. Pour cela vous allez utiliser les pièces que vous voyez ici en les utilisant comme bon vous semble. Nous allons procéder ainsi. D'abord chacun de vous posera à tour de rôle [en partant dans le sens des aiguilles d'une montre] un élément géographique sur la table. Cet élément peut être représenté par un assemblage de pièces ou non. $A$ chaque fois que vous poserez un élément, je vous demande de dire ce que c'est pour que tout le monde le sache. De mon côté, je poserai une étiquette numérotée puis je noterai le lieu sur cette feuille de sorte à ce que je puisse vous rappeler quel été cet élément quand c'est nécessaire. Je vous demande également de porter cette lettre $[A, B, \ldots$ inscrit sur un post-it] sur votre poitrine de sorte à ce que je puisse noter anonymement qui a posé l'élément. Après le premier tour, chacun peut intervenir quand il le souhaite. Simplement, c'est important que les interventions se fassent une à une, de sorte que tout le monde sache ce qui a été ajouté ou modifié sur la table. Sachez que vous pouvez ajouter, déplacer ou modifier un élément, mais vous ne pouvez pas retirer un élément de la table. "

Etape 2 : les participants répondaient individuellement sur une feuille à 13 questions sur le rapport émotionnel à l'espace :

1- Tout d'abord, y-a-t-il un lieu ou un objet qui, en arrivant ici, vous a immédiatement donné l'impression que vous alliez pouvoir travailler agréablement toute cette semaine? Est-ce que ce lieu ou cet objet a changé au cours du temps ? Si oui noter ce nouveau lieu svp.

2- Y-a-t-il un lieu où vous vous êtes senti immédiatement "à votre place " en arrivant ici (un lieu dans lequel vous vous êtes senti immédiatement bien) ? Si oui est-ce que ce lieu ou cet objet a changé au cours du temps ? Si oui noter ce nouveau lieu svp.

3- Y-a-t-il un lieu dans lequel, au contraire, vous avez senti qu'il vous était moins facile « de trouver votre place » en arrivant ici ? Si oui est-ce que ce lieu ou cet objet a changé au cours du temps ? Si oui noter ce nouveau lieu svp.

4- Y-a-t-il un lieu ici que vous évitez la nuit ?

5- Y-a-t-il- un lieu que vous associé au temps qui vous semble le plus fort de ce séjour (pas nécessaire de préciser quel est ce temps fort)?

6- Quel est le lieu qui vous attire le plus ?

7- Quel est le lieu qui vous révulse le plus ?

8- Y-a-t-il un lieu ou un objet ici qui vous rappelle une personne qui vous est chère (pas nécessaire de préciser la personne) ?

9- Au contraire y-t-il un lieu qui vous rappelle une personne avec qui les relations sont difficiles (pas nécessaire de préciser la personne) ?

10- Y-a-t-il un lieu ou un objet ici qui vous évoque le souvenir d'un évènement que vous avez vécu ailleurs (pas nécessaire de préciser l'évènement)?

11- Avez-vous apporté un objet sans lequel vous n'auriez pas pu venir ici ? Si oui, notez où il se trouve actuellement.

12- Y-a-t-il des lieux qui sont sur la reconstruction et pour lesquels vous estimez qu'ils ne font pas partie de ceux dans lesquels nous vivons ensemble depuis vendredi ? 


\section{3- Etes-vous déjà venu à Cerizy ? »}

Etape 3 : les participants procédaient à l'étiquetage émotionnel des lieux à partir de la consigne suivante :

« Revenons maintenant vers la reconstruction (le plateau). Je vous distribue des gommettes. Celles qui sont rouges représenterons les sentiments, les émotions, les affects plutôt négatifs, celles qui sont vertes les affects plutôt positifs et les bleues les affects qui ne sont ni positif ni négatifs ou les deux à la fois. Nous allons reprendre chaque question une à une, sauf les deux dernières, et chacun de vous posera une gommette sur le lieu qui correspond à votre réponse. Mais avant de la posez, je vous demande de noter sur la gommette votre lettre d'identification et le numéro de la question. $\mathrm{Si}$ le lieu manque sur le plateau, vous pouvez l'ajouter et je le numéroterai avec cette gommette (jaune)".

\section{Quelques enseignements en termes d'atouts et de limite sur la méthode}

Quel que soit le groupe, la procédure proposé a permis de rendre compte que certains lieux faisait effectivement l'objet d'une accumulation (ou polarisation) d'affects ayant la même valence (positive ou négative) et que rares étaient les lieux qui n'étaient investis émotionnellement que par une seule personne du groupe.

Cependant, la procédure collective a parfois été vécue par certains comme une contrainte pour évoquer sa conception personnelle du rapport aux lieux (bien que l'étape 2 soit individuelle!), montrant ainsi qu'outre les résistances et les auto-censures parfois rencontrés dans la dimension cognitive de la construction de l'espace géographique (peur de ne pas bien localiser un lieu par rapport aux autres par exemple), nous sommes également face à des résistances quant à une conception sociale des émotions, une résistance globalement formulée sous l'angle d'une dépossession de sa subjectivité (ce qu'un autre atelier du colloque déguisé en visite guidé de Granville a également permis de mettre en évidence lors de débriefing collectif). Ainsi certains participants nous ont précisé que la procédure «ne me permet pas d'exprimer mon approche personnelle ". Notons toutefois que d'autres y trouvent au contraire un avantage : "j'aime bien parce que je n'ai pas à me projeter trop intimement et que mes réponses se fondent dans celles du groupe ». Et plus généralement, la procédure a été vécue comme ludique et agréable.

Une variante de la critique sur la place qu'occupe la dimension personnelle dans la procédure concerne aussi le fait que le déroulé méthodologique repose sur un va-et-vient entre des étapes collectives et individuelles qui peuvent gêner certains participants dans la restitution spatiale des affects. En effet, la spatialisation des affects se fait aux yeux du groupe (étape 3 ) alors qu'ils sont tout d'abord exprimés dans l'intimité de sa propre feuille de notes (étape 2). Autrement dit, le chercheur est face à un dilemme où il doit, soit insister sur le fait que le premier format de réponse sur les affects sera ensuite rendu public, au risque de créer des auto-censures par anticipation de l'expression collective de la dimension spatiale des affects, soit être moins insistant sur la dernière étape du protocole, au risque cette fois de créer une gêne auprès de certains participants au moment venu. Notons que la suppression de la seconde étape, plus individuelle, est finalement une fausse solution, car elle n'aurait pas permis de relever ce problème méthodologique. L'important 
consiste finalement à formuler des questions qui n'obligent pas à rentrer dans l'intimité de la personne (quel est précisément l'objet, la personne, la situation antérieure, la nature précise de l'affect, etc., qui est associé à cette relation entre un affect et un lieu) en mettant l'accent uniquement sur la valence et le lieu des affects.

Dans la même veine, une des difficultés exprimées par les participants en termes de méthode de collecte des émotions liées à l'espace reposait sur une tension en l'objectivation du lieu par l'outil (d'autant que la consigne pouvait aussi inviter à rattacher des émotions à des objets situés) et la subjectivité des émotions. Autrement dit, quand bien même cette dichotomie subjectivité/objectivité est importante à dépasser pour comprendre la construction sociale de l'espace géographique (pour aller vite, on peut dire que l'espace est tout autant dans la matérialité que dans les esprits, mais que la relation entre ces deux dimensions s'organise dans les rapports sociaux), la prégnance de cette division dans les recherches géographiques, comme dans la rationalisation que chacun fait de son rapport à l'espace géographique, permet difficilement de contourner méthodologiquement cette opposition entre subjectif et objectif dans l'expression et la communication des affects géographiques sans provoquer une certaine gêne. Et plus encore, il est important de considérer les tensions liées à des différences d'échelles (par exemple apprécier un objet qui est dans un lieu qui ne l'est pas). Par conséquent, il est important de ne pas amalgamer un objet situé et un lieu dans la manière de présenter l'exercice du recueil des affects.

Quand ce n'est plus l'objectivité/subjectivité qui sont mises en avant mais l'opposition soi/les autres qui constitue le rapport à la méthode de relevé des affects sur les lieux, alors les témoignages semblent converger vers l'expérience d'une articulation étroite plutôt que d'une opposition de ces deux termes, en exprimant que la procédure permet une convivialité dans la construction de l'espace géographique à partir de chaque point de vue, autrement dit tout en partant de soi.

Autre problème : la valence de certaines émotions associées à un lieu n'est pas toujours univoque. Ainsi, un participant précise que la question 5 (Y-a-t-il- un lieu que vous associé au temps qui vous semble le plus fort de ce séjour ?) n'est pas forcément positive. Autrement dit, un temps fort peut aussi être négatif. De plus, en partant des émotions plutôt que de l'espace, on constate que parler d'affects liés à l'espace contribue à la production collective de nouveaux lieux par rapport à la construction collective initiale de l'espace. Et plus généralement, plusieurs témoignages expriment que la nécessaire simplification du rapport affectif aux lieux par cette méthode risque de rendre compte de dimensions affectives appauvries, voire caricaturales, au regard de la complexité des émotions en lien avec les lieux. C'est la raison pour laquelle, les " carte mentales " ne peuvent être étudiées pour elles-mêmes, isolément, mais doivent toujours être associées à un entretien, qui évite la sur-interprétation des " images », et qui permet de restituer la complexité du rapport à l'espace.

Un dernier point important qui a été discuté à propos de cette méthode concerne son caractère suggestif. En effet, et notamment parce que chacun est pris dans une dynamique collective chaque fois qu'il participe ou qu'il répond à une question de la seconde étape, il peut y avoir une sorte d'injonction à associer une émotion ou un affect à un lieu, quand bien même dans son for intérieur nous ne pensons pas nécessaire associer certains type d'émotion à un lieu particulier, et ceci bien que les questions commencent par « $y$-a-t-il un lieu... » plutôt que par « quel est le lieu... ».

Toutes ces limites et ces atouts doivent être replacés dans la problématique de la recherche pour opérer les décisions qui s'imposent et que cet atelier exploratoire a mis en évidence. 